\title{
Correction to: Diffusion and Thermodiffusion of Atmospheric Neutral Gases: a Review
}

\author{
A. V. Pavlov ${ }^{1}$ \\ Published online: 11 March 2020 \\ (c) Springer Nature B.V. 2020
}

\section{Correction to: Surveys in Geophysics (2019) 40:247-276 https://doi.org/10.1007/s10712-019-09522-2}

The original version of this article unfortunately contained misprints. The corrections of these misprints are given below.

On page 259, on the left-hand side of Eq. (76) and on the right-hand side of Eq. (82), there is $v_{q n}$ which should be $v_{q n}$. Corrected Eqs. (76) and (82) should read as follows:

$$
\begin{gathered}
m_{q} \sum_{n(n \neq q)} v_{q n}\left(1-\Delta_{q n}\right)\left(V_{n z}-V_{q z}\right)=k T Y_{q z} \\
2 \cos (\varphi) \Omega<<\sum_{n(n \neq q)} v_{q n} .
\end{gathered}
$$

On page 259, on the right-hand side of the expression (81), there is $v_{q n}\left(1-D_{q n}\right)$ which should be $v_{q n}\left(1-\Delta_{q n}\right)$. The corrected expression (81) should read as follows:

$$
D_{E}(q)=1 / \sum_{n(n \neq q)} X_{n} / D^{*}(q, n)=k T /\left[m_{q} \sum_{n(n \neq q)} v_{q n}\left(1-\Delta_{q n}\right)\right] .
$$

In the first sentence after the expression (81) on page 259 , it is necessary to replace "with" by "with the zero value of the Coriolis term in the expression (77) and".

Publisher's Note Springer Nature remains neutral with regard to jurisdictional claims in published maps and institutional affiliations.

The original article can be found online at https://doi.org/10.1007/s10712-019-09522-2.

A. V. Pavlov

pavlov@izmiran.ru

1 Pushkov Institute of Terrestrial Magnetism, Ionosphere and Radio-Wave Propagation of the Russian Academy of Sciences (IZMIRAN), Moscow, Troitsk, Russia 142190 